\title{
HISTORIA DE UN FRAGMENTO TEXTUAL SOBRE LA EXPLORACIÓN DE LA CHINA. NOTAS LÉXICAS
}

\section{HISTORY OF A TEXTUAL FRAGMENT ON THE EXPLORATION OF CHINA. LEXICAL NOTES}

\author{
Elisa Sartor \\ Università degli Studi di Verona \\ elisa.sartor@univr.it
}

\section{RESUMEN}

Este ensayo se propone estudiar, a través de comprobaciones lexicográficas y en corpus, la historia de algunos términos procedentes de un mismo fragmento textual contenido en dos obras que desempeñaron un papel fundamental en la formación del imaginario chino y oriental en España a finales del siglo XVI. Se trata de Discurso de la navegacion que los Portugueses hazen à los Reinos y Provincias del Oriente, y de la noticia que se tiene de las granderas del Reino de la China (1577) de Bernardino de Escalante y de Historia de las cosas mas notables, ritos y costumbres, del gran Reyno de la China (1585) de Juan González de Mendoza, y de las respectivas traducciones al inglés.

El objetivo de este estudio es ofrecer una reconstrucción contrastiva de la historia de los términos menjui, palo del águila y cayolaque, contenidos en una frase que aparece tanto en el texto de Bernardino de Escalante como en el de Juan González de Mendoza, ambos derivando su origen del tratado portugués de fray Gaspar da Cruz. Se realizará una comparación con las propuestas traductivas respectivamente de J. Frampton y R. Parke.

Además, se proporcionará un ensayo de representación visual de las relaciones entre los tratados y sus fuentes a través de recursos digitales.

Palabras clave: lexicología diacrónica, China, traducción

\begin{abstract}
This essay aims to study -through lexicographical and corpora research - the history of some words extracted from a textual fragment contained in two treatises that played a pivotal role in shaping the Spanish imaginary of China and the East towards the end of the $16^{\text {th }}$ century: Discurso de la navegacion que los Portugueses hazen à los Reinos y Provincias del Oriente, y de la noticia que se tiene de las grandezas del Reino de la Cbina (1577) by Bernardino de Escalante and Historia de las cosas mas notables, ritos y costumbres, del gran Reyno de la China (1585) by Juan González de Mendoza, and their respective English translations.

The objective of this study is offering a contrastive reconstruction of the history of the words menjui, palo del aguila and cayolaque, all of them contained in a sentence that appears both in Bernardino de Escalante's and in Juan González de Mendoza's text; as a matter of fact, both books share a common origin, which is a treatise by Portuguese friar Gaspar da Cruz. We shall also compare the English translations put forward respectively by John Frampton and R. Parke.

Furthermore, this article will provide an attempt at visually representing the relationship between the treatises and their sources through digital resources.
\end{abstract}

Keywords: diachronic lexicology, China, translation 


\section{INTRODUCCIÓN}

Este ensayo se propone estudiar, a través de comprobaciones lexicográficas y en corpus, la historia de algunos términos procedentes de un mismo fragmento textual contenido en dos obras que desempeñaron un papel fundamental en la formación del imaginario chino y oriental en España a finales del siglo XVI. Se trata de Discurso de la navegacion que los Portugueses hazen à los Reinos y Provincias del Oriente, y de la noticia que se tiene de las grandezas del Reino de la China (1577) de Bernardino de Escalante y de Historia de las cosas mas notables, ritos y costumbres, del gran Reyno de la China (1585) de Juan González de Mendoza, y de las respectivas traducciones al inglés.

Nuestro trabajo pretende dar continuación a la investigación empezada en una contribución publicada con anterioridad (Sartor y Dal Maso, 2017), en la que se analizó la traducción inglesa del tratado de Escalante realizada por John Frampton (1579) y la reedición de Thomas Osborne (1745). El presente estudio se centrará en el análisis léxico de un fragmento textual del Discurso, reutilizado posteriormente por Mendoza, actualizando asimismo las reflexiones anteriores de Sartor y Dal Maso (2017) alrededor de las voces empleadas en los textos españoles y en sus traducciones inglesas.

El tratado de Bernardino de Escalante se publicó en 1577 y se compone de dos partes: en la primera se relatan los descubrimientos de los navegadores portugueses y en la segunda se describe China con gran profusión de detalles. Para componer esta obra el religioso de Laredo, que nunca había viajado a China o al Lejano Oriente (Díaz Trechuelo, 1991, p. 15), se basó en dos tratados portugueses, a saber, Ásia de João de Barros — cuyas tres primeras Décadas se habían publicado entre 1552 y 1563 - y el Tractado em que, se contam muito por extenso as cousas da China [...] (1569) de fray Gaspar da Cruz (Vilà, 2013, p. 73). De hecho, el mismo Escalante reconoce sus fuentes y alaba la pericia de los cronistas portugueses: "Iuan de Barros, historiador dotissimo de aquella nacion" (Escalante [1577]1991, 34r).

El Discurso apareció en una época en la que "la curiosidad por el extremo Oriente era un asunto que estaba de candente actualidad [y era] una realidad que afectaba de lleno al interés nacional" (Casado Soto, 1995, p. 62). El libro tuvo un impacto deslumbrante en dos obras publicadas en la década siguiente que se convirtieron en 
verdaderos best sellers de la época: el Theatro de la Tierra Universal (1588) de Abraham Ortelius y la ya mencionada Historia de Mendoza. Desde luego, la aportación de Escalante al Theatro fue reconocida por el propio Ortelius, quien cita al autor cántabro con estas palabras: "De esta región ha escrito un librillo particular Bernardino de Escalante en lengua española, del cual hemos sacado estas cosas siguientes" (apud Díaz Trechuelo, 1991, pp. 27-28); Mendoza, en cambio, omite toda referencia al Discurso. Sin embargo, como demostró Lara Vilà recientemente, "la obra de Escalante, que Mendoza no cita ni una sola vez, es la que procura la estructura general y, a partir de este esqueleto, añade, completa o corrige, en función del material restante del que pudo disponer" (Vilà, 2013, p. 85) ${ }^{1}$.

Además, el tratado de Escalante fue traducido al inglés en 1579 por John Frampton, un hombre de negocios inglés que, después de una larga detención en Sevilla por parte de la Inquisición, volvió a Londres y desempeñó una importante labor traductora (Wroth, 1954; Beecher, 2006). El tratado de Mendoza (1585) fue traducido al inglés por R. Parke ${ }^{2}$ tan solo tres años después de su primera edición española; en efecto, Beecher (2006, p. 331) y Wroth (1954, pp. 311-313) coinciden en señalar que el interés comercial despertado por las exploraciones hacia China en aquella década era máximo: fue justamente al principio de la década de los ochenta cuando zarpó la expedición de Jackman y Pet para buscar una ruta marítima hacia la China por el Ártico (1580).

\section{METODOLOGÍA Y OBJETIVOS}

Las cinco obras a partir de las cuales se ha espigado el fragmento textual objeto de estudio son el Tractado (1569) del portugués fray Gaspar da Cruz, el Discurso de Bernardino de Escalante (1577) junto con la traducción inglesa de John Frampton $A$ discourse of the nanigation which the Portugales doe make to the Realmes and Prouinces of the East partes of the worlde [...] (1579), y la Historia (1585) de Juan González de Mendoza con su traducción al inglés por R. Parke, The Historie of the great and mightie kingdome of China

\footnotetext{
1 Las distintas fuentes utilizadas por Mendoza han sido estudiadas por numerosos investigadores, entre ellos Santos Rovira (2006), que destaca la influencia del manuscrito Relación de el viaje que hezimos en China [... (1580) de fray Agustín de Tordesillas.

${ }^{2}$ No se ha podido encontrar el nombre de pila del traductor, R. Parke.
} 
(1588). Para tener acceso a las ediciones prínceps se han consultado los repositorios en línea de numerosas instituciones, entre ellas la Biblioteca Digital Hispánica de la Biblioteca Nacional de España y la Biblioteca Virtual del Consejo Superior de Investigaciones Científicas (CSIC), la Biblioteca Nacional Digital de la Biblioteca Nacional de Portugal, además de Early English Books Online (EEBO), Iberian Books (University College Dublin), Europeana y Google Books. De cada libro se ha usado una versión digitalizada de la edición original, excepto en el caso del Discurso de Escalante, disponible en una edición facsímil de 1997 de la Universidad de Cantabria: en concreto, se ha cotejado la versión digital de la Historia de Mendoza conservada en la Biblioteca Casanatense de Roma, los ejemplares digitalizados de las traducciones inglesas de Escalante y de Mendoza guardadas en la Huntington Library; por último, el tratado de fray Gaspar da Cruz de la Biblioteca Nacional de Portugal.

Las herramientas lexicográficas utilizadas para llevar a cabo el análisis léxico consisten en los diccionarios académicos y no académicos consultables en el Nuevo tesoro lexicográfico de la lengua española (NTLLE), además del Nuevo tesoro lexicográfico del español (s. XIV-1726) de Nieto y Alvar Ezquerra (2007) y el Diccionario crítico etimológico castellano e hispánico (1980-1991) de Corominas y Pascual. Naturalmente se han consultado los tres diccionarios históricos de la RAE: el primero, incluido ya en el NTLLE, el segundo (DHLE 1960-96), que también es un proyecto truncado, y el actual que, como es notorio, es una obra en fase de realización. Estos últimos no han devuelto resultados debido al hecho de que las palabras estudiadas no se recogen en la nomenclatura parcial de estas obras incompletas. Se ha utilizado el Corpus diacrónico del español (CORDE) de la Real Academia Española con el propósito de comprobar las ocurrencias textuales de los términos objeto de estudio. En cuanto a la lengua inglesa, la referencia principal ha sido el Oxford English Dictionary (OED). Se ha tomado en consideración también una edición facsímil de The Boke of Englysshe, and Spanisshe (1554), siendo una de las obras lexicográficas al alcance de los traductores ingleses. Sin embargo, su consulta no ha proporcionado datos útiles para nuestra investigación.

El objetivo de este estudio es el de ofrecer una reconstrucción contrastiva de la historia de los términos menjui, palo del águila y cayolaque, contenidos en una frase que 
aparece tanto en el texto de Bernardino de Escalante como en el de Juan González de Mendoza, ambos derivando su origen del tratado portugués de fray Gaspar da Cruz. Se realizará una comparación con las propuestas traductivas respectivamente de John Frampton y R. Parke. Para este trabajo se ha decidido prescindir del análisis de las numerosas traducciones a otros idiomas del tratado de Mendoza, cuya enorme fortuna y circulación en Europa ${ }^{3}$ merecen un estudio pormenorizado.

Esta compleja red de reediciones y traducciones de los tratados que nos ocupan y su rico entramado textual, que abarca numerosos países europeos y en el que se profundizará en trabajos futuros, se podrían representar visualmente gracias a un mapeo realizado a partir de recursos digitales, del cual se ofrece un primer ensayo simplificado, puesto que se basa únicamente en los cinco textos acotados en esta investigación. Para ello, se ha realizado un mapa a través de la plataforma de acceso libre Omeka/Neatline, una herramienta basada en la georreferenciación que permite presentar y describir gráficamente fenómenos de distinta tipología ${ }^{4}$. En el caso concreto, gracias al plug-in Waypoints se han producido unas fichas que resumen las relaciones textuales entre los volúmenes estudiados.

\footnotetext{
${ }^{3}$ Da fe de ello Vilà (2013, pp. 92-93) en una tabla que recoge todas las ediciones publicadas en los siglos XVI y XVII.

${ }^{4}$ Para ahondar en el debate que media entre los problemas relacionados con las técnicas de mapeo y las humanidades digitales, hasta llegar a los últimos desarrollos de GIS (sistemas de información geográfica) en el ámbito de las humanidades espaciales, se remite a Monmonier (1996), Bodenhamer, Corrigan y Harris (2010), Eide (2015) y Sartor (2019). El programa Omeka/Neatline se ha podido utilizar gracias al apoyo técnico del Departamento de Humanidades digitales de la Universidad de Colonia.
} 


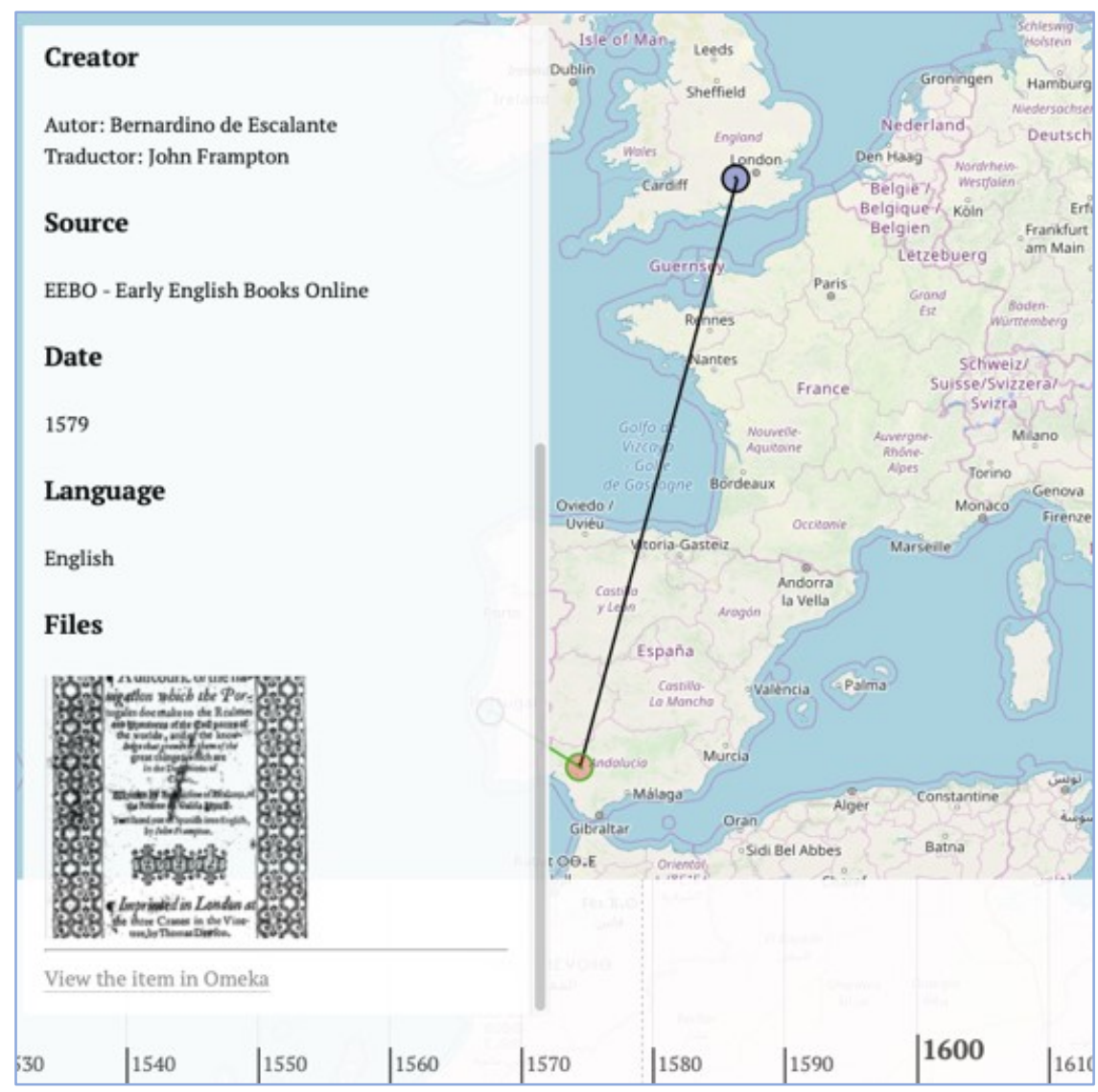

Figura I. Ejemplo de ficha bibliográfica Dublin Core en Neatline

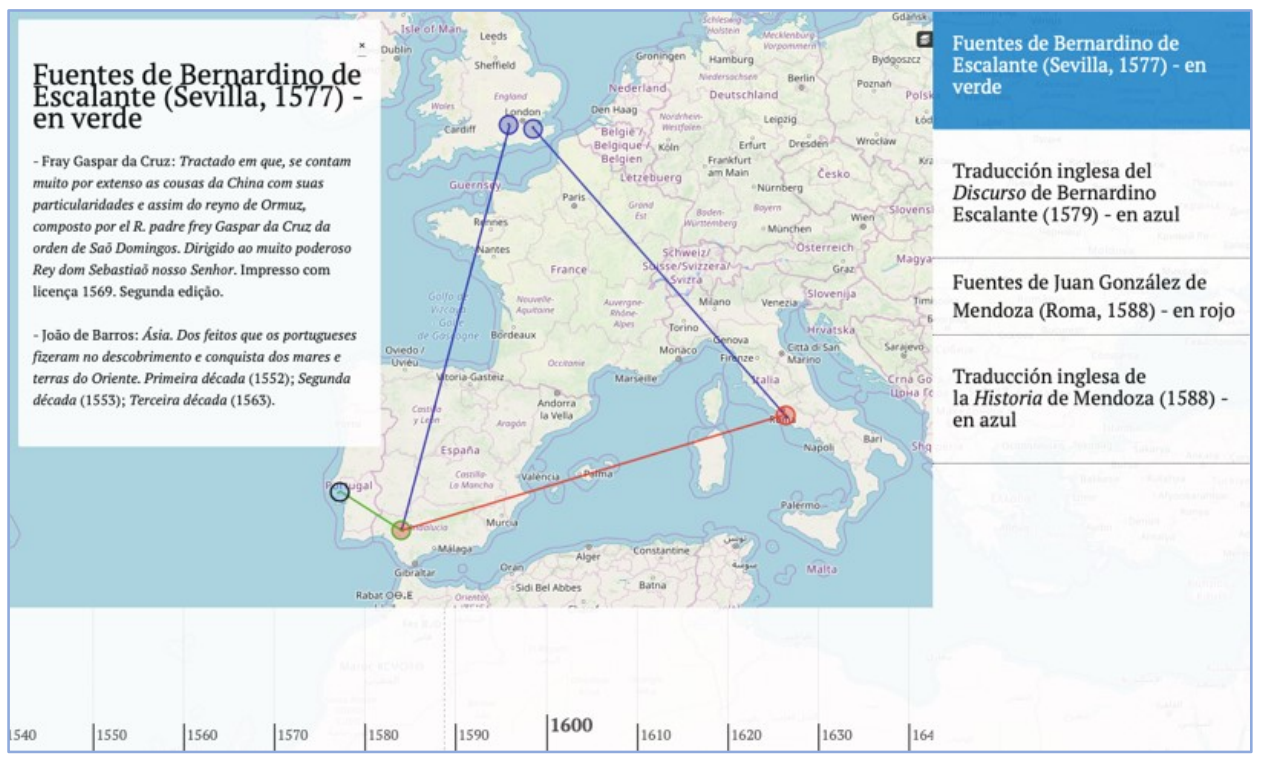

FIgURA II. Ejemplo de representación visual en Neatline de las relaciones entre los tratados y sus fuentes en el corpus acotado 
En las imágenes arriba se busca dar cuenta del proceso de creación - todavía in fieride una representación visual de las relaciones textuales en el corpus estudiado. La figura 1 ejemplifica una ficha bibliográfica en Omeka realizada según el estándar Dublin Core, mientras que la figura 2 hace uso del plugin Waypoint para ilustrar los periplos del fragmento textual objeto de estudio a través de las fuentes y de las traducciones: del Tratado de fray Gaspar da Cruz al Discurso de Bernardino de Escalante; de este a la traducción inglesa de Frampton pero también a la Historia de Mendoza y a la traducción inglesa de Parke. Las líneas de colores empleadas reflejan el cambio de idioma intervenido: del portugués al español en verde y del español al inglés en morado; el trazo rojo, en cambio, da constancia de la reelaboración del libro de Escalante en el tratado de Mendoza. Aunque de limitado alcance debido al corpus restringido que se ha analizado en esta ocasión, el mapa creado con Neatline da fe del potencial de esta herramienta para describir visualmente datos bibliográficos y editoriales.

\section{ANÁLISIS: DE LOS TEXTOS A LA LEXICOGRAFÍA}

Debido a la tipología misma de la obra, el léxico de los dos tratados en lengua española es muy variado. Así pues, al tratarse de relaciones de viaje, es posible encontrar tanto vocabulario de la navegación como de la botánica y la zoología, necesarios a la hora de proporcionar una descripción geográfica del territorio explorado, sin olvidar la terminología relacionada con la arquitectura y los rituales, que sirve para ofrecer un acercamiento etnográfico a la realidad acotada. Además, Mendoza profundiza también, entre otros aspectos, en el arte de la imprenta y el uso de la artillería en China, ampliando aún más la pluralidad de ámbitos léxicos presentes en su texto.

Para la selección de los términos objeto de estudio, se han buscado voces procedentes de un dominio de especialidad que hasta la fecha no ha sido estudiado ${ }^{5}$. Con el objetivo de facilitar la búsqueda de las correspondencias en los textos acotados, se han extraído tres unidades terminológicas dentro de una misma oración, lo que ha permitido identificar los fragmentos útiles para el análisis de manera certera y unívoca.

\footnotetext{
${ }^{5}$ En un estudio anterior (Sartor-Dal Maso, 2017) se efectuó un primer acercamiento lexicológico al tratado de Escalante centrado, en su mayor parte, en el ámbito de la zoología.
} 
Concretamente, los términos se hallan en un capítulo que delinea la religión de los chinos y se originan en el vocabulario de la botánica, refiriéndose a sustancias olorosas empleadas en los rituales.

Los términos seleccionados para el análisis léxico - menjui, palo del águila y cayolaqueproceden de una frase del tratado de Bernardino de Escalante ([1577] 1991, 89v), "Ofrece esta gente à las mañanas y à las tardes en sus templos incienso, menjui, palo del Aguila, y Cayolaque, y otras pastas de diferentes y suaves olores [...]", que luego confluyó con variaciones mínimas en el tratado de Juan González de Mendoza (1585, p. 46): "Ofrecen a las mañanas, y tardes a los Ydolos encienso, menjui, palo del aguila, y cayolaque que es muy oloroso, y otras pastas de differentes, y suaves olores". Como ya se ha adelantado, la fuente de Escalante fue el texto del portugués fray Gaspar da Cruz (1569, cap. XXVII): “offerecemlhes Incenso \& Benjoy, Aguilla, \& outro pao que chaman Cayo, Laque \& outros cheiros". Es oportuno aclarar que, pese a que el autor portugués escribiera cayolaque por separado, en otro punto del texto el lema aparece unido gráficamente: "\& de um pao cheiroso, que chaman Cayolaque, \& de sandalo" (Da Cruz, 1569, cap. XI).

El arabismo menjui despierta cierto interés desde el punto de vista ortográfico por la vacilación del grafema inicial $<\mathrm{m}>/<\mathrm{b}>$. En general, la voz menjui remite a benjui $\mathrm{y}$, a partir de la segunda mitad del siglo XVIII, la lexicografía tanto académica como no académica se decanta por la forma aguda (menjuí desde la edición del diccionario de la Real Academia de 1780 y benjui desde la de 1770). El diccionario de Autoridades de 1726 proporciona la siguiente definición: Benjui. "Liquór o goma que destíla el arbol llamado Laserpicio. [...] Vulgarmente le llaman Menjui; pero su nombre verdadero es Benjui". A partir de 1817 se define como 'bálsamo aromático', lo que permanece estable en la lexicografía hasta hoy: "Benjuí. Bálsamo aromático que se obtiene por incisión en la corteza de un árbol del mismo género botánico que el que produce el estoraque en Malaca y en varias islas de la Sonda" (DLE). Terreros (1786), por ejemplo, añade la siguiente explicación: "De esta resina hai tres especies todas diversas de la Asafétida, V. Asa". El diccionario de la editorial Gaspar y Roig (1853) aclara que se "estrae del estoraque benjuí, árbol orijinario de las islas de la Sonda", indicación geográfica que se 
mantiene en la definición actual de Diccionario de la lengua española. El diccionario etimológico de Corominas y Pascual, por su parte, recoge solamente benjuí, vocablo de procedencia árabe con significado de 'incienso de Sumatra', documentado en castellano a partir del año $1438^{6}$. Los resultados en CORDE son numerosos con ambas grafías y confirman la preferencia por la variante aguda y con $<b>$, coherentemente con los resultados de las pesquisas lexicográficas.

La lexía palo del águila aparece en la lexicografía española a partir del diccionario de la Real Academia de 1780, cuando entra en la definición de aspalato: "Llámase tambien por algunos palo del aguila, ó palo de la rosa". En el diccionario académico de 1803 se desdobla en dos voces distintas: palo de águila, "Lo mismo que aloe árbol, que es más usado" y palo del águila, "Lo mismo que aspalato, que es más usado". En la edición de 1822 aparecen dos entradas por palo del águila, que remiten a palo áloe y aspalato respectivamente, así como en el diccionario de Núñez de Taboada (1825). Desde el diccionario académico de 1832 se introduce la definición de ‘arbusto' y la sinonimia con alarguez $^{7}$, que se mantiene en los diccionarios de Salvá (1843), de Domínguez (1853) y de la editorial Gaspar y Roig (1855); sin embargo, a finales del siglo Zerolo (1895) remite a palo de áloe y la Real Academia (1899) proporciona la siguiente definición: "Madera de un árbol de la familia de las terebintáceas, algo parecida al palo áloe". Con el diccionario académico de 1914 se inaugura un cambio que permanecerá vigente hasta la edición de 1992: palo del águila pasa a formar parte de la definición de palo áloe: "Madera del agáloco, muy resinosa, amarga y purgante como el acíbar, empleada en farmacia y como sahumerio en Oriente. || Madera del calambac, muy parecida a la anterior". No es muy distinta la actual definición ofrecida por el DLE: "Madera de un árbol de la familia de las timeleáceas, algo parecido al palo áloe".

\footnotetext{
${ }^{6}$ El Nuevo tesoro de Nieto y Alvar Ezquerra (2007) ofrece una primera atestación de benjuí más tardía, siendo extractada del Universal vocabulario de Palencia (1490).

7 Tanto la definición de aspálato como la de alarguez permanecen invariadas en la lexicografía académica desde el diccionario académico de 1884: "Nombre dado a varias plantas espinosas parecidas a la retama y a algunas maderas olorosas" y "Nombre que se ha dado a varias plantas espinosas, especialmente al agracejo y al aspálato", respectivamente (DLE). En la actualidad aspálato y alarguez designan, entre otras, la planta Berberis vulgaris de la familia de las Berberidaceae; Aspalathus, en cambio, es un género que pertenece a la familia de las Fabaceae.
} 
Palo del águila se recoge en Nieto y Alvar Ezquerra (2007), que proporciona una traducción procedente del diccionario de Pedro Seguin de 1636: "palo de águila, bois de senteur'. La única referencia en CORDE está sacada del tratado del proprio Mendoza (1585, p. 380): “También los japoneses llevan a vender allí su plata, y los del Reino de Siam muchas cosas muy curiosas, en especial clavo y pimienta de las Islas Malucas, y los de Borneo mucho sándalo y nuez moscada; los de Java y Pegu, el palo del águila [...]”. En la lexicografía tanto académica como no académica, el significado de palo del águila oscila entre el de arbusto y de árbol, decantándose por este último a partir del siglo XX.

Cabe señalar que palo como núcleo de compuestos sintagmáticos con un modificador (tanto un adjetivo como la preposición de seguida por un sustantivo) es muy productivo en la formación de fitónimos: se destaca, entre otros, palo amarillo, palo blanco, palo rojo, palo borracho, palo dulce, palo santo, palo cochino, palo de balsa, palo de Fernanbuco, palo de jabón, palo de las Indias, etc.

El tercer término en que se centra nuestro análisis, cayolaque, tuvo una trayectoria breve y singular, que no dejó rastro en los diccionarios académicos. Aparece, en cambio, como también confirman Nieto y Alvar Ezquerra (2007), en varios diccionarios bilingües y multilingües de los siglos XVII y XVIII, como el de Oudin (1607, francés-español), “une sorte de parfum”, el de Vittori (1609, francés-italianoespañol), "une sorte de parfum des Indiens, una sorte di perfumo de Indiani”, el de Minsheu (1617, español-latín-inglés), “a kinde of perfume”, el de Franciosini (1620, español-italiano), "Una sorte di profummo Indiano", y el de Stevens (1706, españolinglés), "a sort of Perfume".

El único diccionario monolingüe de la lengua general que lo registra es el de Terreros (1786 [1767]): “Cayoláque: cierto perfume”; sin embargo, el diccionario de Terreros lematiza también cayelác, "del fr. Cayelac, madera olorosa del Reino de Siám, donde, y en la China, sirve de incienso para los Templos de los Gentiles”, como harán, con una definición muy parecida, también los suplementos del diccionario de Domínguez (1853 y 1863), el diccionario de la editorial Gaspar y Roig (1853) y el de Zerolo (1895). En la lexicografía sucesiva cayolaque no se registra, excepto naturalmente 
en el Diccionario histórico (1933-1936), que cita el texto de Mendoza y las entradas lexicográficas de Oudin y Terreros para apoyar su definición, "Perfume de las Indias Orientales". El diccionario de Corominas ofrece una definición de cayolaque bajo la voz badulaque, aclarando que "la semejanza con badulaque parece ser en este caso un producto del azar”. La definición es coherente con la lexicografía anterior y esclarece la procedencia botánica de esta sustancia: "nombre de un perfume de las Indias Orientales, propiamente nombre de la Myristica iners ${ }^{8}$ de donde se extrae este perfume, y compuesto del malayo kayu 'árbol' y laka 'laca"'. CORDE también devuelve como único resultado el texto de Mendoza. Cayolaque es sin duda una voz de uso muy restringido y de corta vida, un neologismo efímero, cuya difusión en los diccionarios bilingües y multilingües se debió a la fortuna de las traducciones de la Historia a otras lenguas europeas.

La traducción inglesa de cayolaque, sin embargo, ha llegado hasta hoy en día. La búsqueda en el Oxford English Dictionary, en efecto, nos devuelve el lema cayolac, con las variantes cayelac y cayolaque y la marca diacrónica obsolete. La primera atestación recogida por $O E D$ es justamente la traducción al inglés del tratado de Mendoza realizada por Parke en 1588: "Euerie morning and euening they do offer unto their Idolles, frankensence, beniamin, wood of aguila and cayolaque [the which is maruelous sweete, and other gummes of sweet and odoriferous smels]" (Mendoza, 1588, p. 41). Oportunamente, los compiladores de $O E D$ añaden información etimológica que coincide grosso modo con la de Corominas: "Malay kayu wood, laka the wood of Myristica iners (or? Tanarius major) used as incense". La última entrada (1859, procedente de Simmonds) asegura tratarse de "an aromatic wood obtained in Siam".

En 1579 John Frampton, el traductor del tratado de Bernardino de Escalante, había obviado al problema con cierta elegancia, omitiendo toda referencia a este lema problemático y sustituyéndolo con una paráfrasis: "These people do offer in the Mornings and Euenings in their Temples, Incense, Beniamin, the wood of the Eagle, and other things of different and sweet smels"(Escalante, 1579, 45r) por "Ofrece esta

\footnotetext{
${ }^{8}$ Es un árbol tropical de la familia Myristicaceae, perteneciente al orden de las Magnoliales. De la misma familia es muy conocida la especie Myristica fragrans, de la que se obtiene la nuez moscada.
} 
gente à las mañanas y à las tardes en sus templos incienso, menjui, palo del Aguila, y Cayolaque, y otras pastas de diferentes y suaves olores [...]" (Escalante, 1577, 89v) ${ }^{9}$.

Mientras que la traducción de menjui no supuso dificultad alguna ni por parte de Frampton ni de Parke, siendo el lema beniamin (en la actualidad con grafía benjamin, 'gum benzoin') atestado en la lengua inglesa desde el siglo XV (OED), merecen un breve comentario las propuestas traductivas de ambos del sintagma palo del águila, a saber, wood of the Eagle y wood of Aguila, respectivamente. Ninguna de las dos acuñaciones prosperaría en la lexicografía inglesa, que recoge en cambio eagle-wood como compuesto endógeno ("Formed within English, by compounding", OED) a partir del siglo XVII. Sin embargo, reconocen los compiladores del diccionario histórico inglés el papel que pudo tener el sintagma portugués pau de águila ${ }^{10}$ junto con el lema holandés arendshout en la formación del compuesto inglés, con el significado de "The aromatic resinous heartwood of the South-East Asian tree Aquilaria malaccensis and certain other trees of the genera Aquilaria and Gyrinops" (OED). Bajo el lema agila se cita otra vez la traducción de Parke de la frase que nos viene ocupando, lo que nos lleva a ensalzar, una vez más, el impacto que tuvo la circulación del tratado de Mendoza en la Europa del siglo XVII.

A este respecto, unas búsquedas preliminares en los diccionarios históricos del francés y el italiano han puesto de relieve el hecho de que estos términos pasaron a las respectivas tradiciones lexicográficas en manera desigual: mientras que menjui/benjuí está atestado tanto en Rey (2010) como en Battaglia (1984) (benjoin y bengiui/ benzoino, respectivamente), solo este último recoge palo d'aquila ("legno ricavato all'aloe Aquilaria agallocha, agalloco") y ninguno de los dos incluye una posible traducción de cayolaque.

\footnotetext{
${ }^{9}$ La omisión -además de "una reformulación más ambigua de los sintagmas para disimular su escaso entendimiento del término" (Sartor-Dal Maso, 2017, pp. 243-244) - es una estrategia que Frampton emplea en varias ocasiones a lo largo de su traducción del tratado; sin embargo, un análisis detenido de las técnicas utilizadas por Frampton y Parke en relación con las prácticas traductológicas vigentes en el siglo XVI excede a los límites de esta contribución.

${ }^{10}$ En lo que se refiere al compuesto que OED cita como pau de águila, las comprobaciones lexicográficas realizadas en el diccionario de Antonio de Morais Silva de 1789 confirman esta grafía. En efecto, Silva recoge dos lemas, uno relacionado con la ornitología y el otro con la botánica: aguia ("s. f. ave de rapiña, e he mais nobre de todas") y aguila ("s. m. lenho aromatico de Asia, que he o samo, ou branco do aloes"). Es este segundo lema el que pasa a formar parte del compuesto páo de Aguila, que aparece bajo la voz páo con significado de 'lenho'.
} 
En el Dictionnaire de Littré (1872-1877), además de benjoin, aparece también bois d'aigle con la siguiente definición: “ou bois de garo, nom donné à différents arbres des Indes et des Moluques, appartenant aux genres agallochum, aquilaria”.

\section{CONCLUSIONES}

Este recorrido por algunas de las obras que marcaron la percepción de la China en España y en Europa pretende ser un acercamiento desde la vertiente de los estudios diacrónicos del léxico a una materia de gran complejidad. Nuestra aproximación ha puesto de relieve la interconexión entre el Tractado de fray Gaspar da Cruz, el Discurso de Bernardino de Escalante y la Historia de Juan González de Mendoza, de la que se ha proporcionado una representación visual con el programa Omeka y su plugin Neatline. A través de una búsqueda lexicográfica se ha intentado reconstruir la historia de unas palabras que aparecen en un mismo fragmento textual, tanto en castellano como, en clave contrastiva, en inglés, para comprobar las soluciones adoptadas por los traductores de Escalante y Mendoza: J. Frampton y R. Parke, respectivamente.

De estas consultas cabe destacar la temprana y coherente atestación de menjui/ benjuí, pese a las oscilaciones en cuanto a su forma gráfica; el compuesto palo del águila, en cambio, además de variantes morfológicas, presenta variaciones en cuanto al significado, siendo relacionado con arbustos -aspálato y alarguez- y con el árbol agáloco. Por último, cayolaque es un neologismo efímero y de uso limitado que se recoge principalmente en los diccionarios bi- y multilingües, debido a la circulación de las traducciones europeas del exitoso tratado de Mendoza.

Los logros de los traductores ingleses, por su parte, han sido dispares: por un lado, pudieron encontrar un lema correspondiente para menjui sin la menor dificultad; por otro, no consiguieron acuñar una solución satisfactoria para palo del águila. Y ante el reto traductivo representado por cayoalque, Frampton decidió tirar la toalla, mientras que el cayolac de Parke se conserva hasta hoy en día en el OED, aunque con marca diacrónica de término obsoleto.

El sondeo de las entradas en la lexicografía francesa e italiana es prometedor en cuanto a las perspectivas futuras de investigación abiertas por este breve trabajo. Se 
precisa un estudio terminológico contrastivo en un corpus textual más amplio que incluya las traducciones a las demás lenguas europeas —acompañado de una representación gráfica sintética realizada a través de medios digitales — con el afán de proporcionar una visión abarcadora de la circulación de ideas y de los mecanismos léxicos y neológicos que permitieron acuñar los lemas correspondientes.

\section{BIBLIOGRAFÍA}

\section{FUENTES PRIMARIAS}

Barros, J. de (1552). Ásia. Dos feitos que os portugueses fizeram no descobrimento e conquista dos mares e terras do Oriente. Primeira década [edición facsímil. Lisboa, Imprensa Nacional-Casa da Moeda, 1988].

Barros, J. de (1553). Ásia. Dos feitos que os portugueses fiveram no descobrimento e conquista dos mares e terras do Oriente. Segunda década [edición facsímil. Lisboa, Imprensa Nacional-Casa da Moeda, 1988].

Barros J. de (1563). Ásia. Dos feitos que os portugueses fizeram no descobrimento e conquista dos mares e terras do Oriente. Terceira década [edición facsímil. Lisboa, Imprensa Nacional-Casa da Moeda, 1992].

Cruz, G. da (1569). Tractado em que, se contam muito por extenso as cousas da China com suas particularidades e assim do reino de Ormuz, composto por el R. padre Frey Gaspar da Cruz da Ordem de Saõ Domingos. Dirigido ao muito poderoso Rey dom Sebastiaõ nosso Senhor. Impresso com licença 1569.

Escalante, B. de ([1577] 1991). Discurso de la navegacion que los Portugueses hazen à los Reinos y Provincias del Oriente, y de la noticia que se tiene de las grandezas del Reino de la Cbina. Edición facsímil de la princeps, por L. Díaz Trechuelo. Cantabria: Universidad de Cantabria, Ayuntamiento de Laredo.

Escalante, B. de (1579). A discourse of the nanigation which the Portugales doe make to the Realmes and Proninces of the East partes of the worlde, and of the knowledge that growes by them of the great thinges which are in the dominions of China. Written by Bernardine of Escalanta, of the Realme of Galisia, priest. Translated out of Spanish into English by Iohn Frampton. London: Thomas Dawson.

González de Mendoza, J. (1585). Historia de las cosas mas notables, ritos y costumbres, del gran Reyno de la China, sabidas assipor los libros delos mesmos Chinas, como por relación de Religiosos y otras personas que an estado en el dicho Reyno [...]. Roma: a costa de Bartholome Grassi; en la Stampa de Vincentio Accolti.

González de Mendoza, J. (1588). The Historie of the great and mightie kingdome of China, and the situation thereof: Togither with the great riches, huge Citties, politike gouernment, and rare inuentions in the same. Translated out of Spanish by R. Parke. London: Printed by I. Wolfe for Edward White.

Ortelius, A. (1588). Theatro de la Tierra Universal de Abraham Ortelio. Anvers: C. Plantino.

Osborne, T. (1745). A collection of Voyages and Travels, constisting of Authentic Writers in our own Tongue, which have not before been collected in English, or have only been abridged in other Collections. And continued with Others of Note, that have published Histories, Voyages, Travels, Journals or Discoveries in other Nations and Languges, relating to Any Part of the Continent of Asia, Africa, America, Europe, or the Islands thereof [...]. Londres: Thomas Osborne. 


\section{FUENTES SECUND ARIAS}

Beecher, D. (2006). The Legacy of John Frampton: Elizabethan Trader and Translator, en Renaissance Studies, 20 (3), pp. 320-339.

Bodenhamer, D. J., Corrigan, J., Harris, T. M. (Eds.) (2010). The Spatial Humanities: GIS and the Future of Humanities Scholarship. Bloomington: Indiana University Press.

Casado Soto, J. L. (1995). Discursos de Bernardino de Escalante al Rey y sus Ministros (1585-1605). Santander: Universidad de Cantabria, Ayuntamiento de Laredo.

Díaz Trechuelo, L. (1991). La obra de Bernardino de Escalante. En L. Díaz Trechuelo (Ed.), Discurso de la navegacion que los Portugueses hazen à los Reinos y Provincias del Oriente, y de la noticia que se tiene de las grandezas del Reino de la China. Sevilla. Edición facsímil de la princeps. Cantabria: Universidad de Cantabria, Ayuntamiento de Laredo, pp. 15-55.

Eide, Ø. (2015). Media Boundaries and Conceptual Modelling: Between Texts and Maps. Basingstoke: Palgrave Macmillan.

Monmonier, M. (1996). How to Lie with Maps. $2^{\mathrm{a}}$ ed. Chicago: University of Chicago Press.

Santos Rovira, J. M. (2006). Estudio histórico-filológico de la crónica del viaje a China de fray Agustín de Tordesillas, en eHumanista, 7, pp. 115-126.

Sartor, E. (2019). Corpus textuales y mapas digitales: un estudio de la circulación de Arte de los metales (1640) de Álvaro Alonso Barba en Europa y América durante la Edad Moderna a través de las humanidades espaciales. En M. De Beni (Ed.), Imagen y discurso técnico-científico en español. Miradas interdisciplinarias, Colección Pliegos Hispánicos. Mantova: Universitas Studiorum, pp. 197-219.

Sartor, E. y Dal Maso, E. (2107). Fortuna y traducción de las relaciones acerca del Lejano Oriente en España y Europa. Estudio textual y léxico sobre el Discurso de la navegacion que los Portugueses hazen à los Reinos y Provincias del Oriente, y de la noticia que se tiene de las grandezas del Reino de la China (1577) de Bernardino de Escalante y sus versiones inglesas (1579; 1745). En F. del Barrio de la Rosa (Ed.), Palabras Vocabulario Léxico. La lexicología aplicada a la didáctica y a la diacronía. Colección VenPalabras. Estudios de lexicología española. Venezia: Edizioni Ca' Foscari, pp. 237-249.

Vilà, L. (2013). La Historia del Gran Reino de la China de Juan González de Mendoza. Hacia un estudio de las crónicas de Oriente en la España del Siglo de Oro, en Boletín Hispánico Helvético, 21, pp. 71-97.

Wroth, L. C. (1954). An Elizabethan Merchant and Man of Letters, en Huntington Library Quarterly, 17, 4, pp. 299-314.

\section{CORPUS LEXICOGRÁFICOS, BANCOS DE DATOS, RECURSOS ELECTRÓNICOS}

Alston, R. C. (Ed.) ([1554] 1971). The Book of English and Spanish. Menston: The Scolar Press Limited.

Battaglia, S. (1984). Grande dizionario della lingua italiana. Torino: UTET.

Biblioteca Nacional de España. Biblioteca Digital Hispánica [en línea] http://www.bne.es/es/Catalogos/BibliotecaDigitalHispanica [21-01-2019].

Biblioteca Nacional de Portugal. Biblioteca Nacional Digital [en línea] http://purl.pt/index/geral/PT/index.html [21-01-2019].

CSIC, Consejo Superior de Investigaciones Científicas. Biblioteca Virtual [en línea] http://bibliotecas.csic.es/biblioteca-virtual [21-01-2019]. 
Corominas, J. y Pascual, J. A. (1980-1991). Diccionario crítico etimológico castellano e hispánico. Madrid: Gredos.

Littré, É. (1872-1877). Dictionnaire de la langue française. Paris: Libraire de l'Hachette [en línea] http://artflproject.uchicago.edu/node/17 [21-01-2019].

EEBO = Early English Books Online [en línea] http://eebo.chadwyck.com/home [21-01-2019].

Europeana [en línea] http://www.europeana.eu/portal/es [21-01-2019].

Google Books [en línea] https://books.google.com [21-01-2019].

NDHE. Real Academia Española: Nuevo diccionario bistórico del español [en línea] http://web.frl.es/DH/org/login/Inicio.view [21-01-2019].

Nieto Jiménez, L. y Alvar Ezquerra, M. (2007). Nuevo tesoro lexicográfico del español (s. XIV-1726). Madrid: Editorial Arco Libros.

OED. Oxford English Dictionary Online [en línea] http://www.oed.com/ [21-01-2019].

Real Academia Española (1960-1996). Diccionario bistórico de la lengua española [en línea] http://web.frl.es/DH.html [21-01-2019].

Real Academia Española (2001). Nuevo tesoro lexicográfico de la lengua española. Madrid: Espasa. [en línea] http://www.rae.es/recursos/diccionarios/diccionarios-anteriores-1726-1992/nuevo-tesorolexicografico $[21-01-2019]$.

Real Academia Española. Banco de datos (CORDE) [en línea]. Corpus diacrónico del español http://corpus.rae.es/cordenet.html [21-01-2019].

Rey, A. (2010). Dictionnaire historique de la langue française. Paris: Le Robert.

Silva, A. de Morais. (1789). Diccionario da lingua portugueza composto pelo padre D. Rafael Bluteau, reformado, $e$ accrescentado por Antonio de Moraes Silva natural do Rio de Janeiro, tomo primeiro A-K. Lisboa na Officina de Simão Thaddeo Ferreira [en línea] https://archive.org/details/diccionariodalin00mora/page/n4.

Silva, A. de Morais. (1789). Diccionario da lingua portugueza composto pelo padre D. Rafael Bluteau, reformado, $e$ accrescentado por Antonio de Moraes Silva natural do Rio de Janeiro, tomo segundo L-Z. Lisboa na Officina de Simão Thaddeo Ferreira len línea] https://books.google.it/books?id=4FkSAAAAIAAJ\&printsec=frontcover\&source=gbs ge summ ary $\mathrm{r} \& \mathrm{cad}=0 \#_{\mathrm{v}}={ }_{\text {onepage } \& \mathrm{q} \& \mathrm{f}=\text { false. }}$.

University College Dublin. Iberian Books [en línea] http://iberian.ucd.ie/ [21-01-2019]. 